MABOKO, MM; PLOOY, CP; CHILOANE, S. 2017. Yield of determinate tomato cultivars grown in a closed hydroponic system as affected by plant spacing. Horticultura Brasileira 35: 258-264. DOI - http://dx.doi.org/10.1590/S0102-053620170217

\title{
Yield of determinate tomato cultivars grown in a closed hydroponic sys- tem as affected by plant spacing
}

\author{
Martin M Maboko; Christian P Du Plooy; Silence Chiloane
}

Agricultural Research Council, Vegetable and Ornamental Plants, Pretoria, South Africa; mmaboko@arc.agric.za; iduplooy@arc.agric. za; chiloanes@arc.agric.za

\begin{abstract}
In South Africa there are no established spacing recommendations for determinate tomato cultivar production under closed hydroponic systems. This study was conducted to determine yield and quality of hydroponically grown determinate tomato cultivars at different plant densities. Four determinate tomato cultivars (Dynimo, Mariana, Mion and Sama) were subjected to four plant densities $(10,16,20$ and 25 plants $/ \mathrm{m}^{2}$ ) during the spring/summer and summer/fall seasons. Experimental layout was a randomized complete block design in a factorial scheme of four plant spacing $\mathrm{x}$ four cultivars with three replicates. There were no significant differences in marketable yield between the cultivars tested during the spring/summer and summer/ fall seasons. Cultivar Mion showed higher incidence of blossom end rot, while Mariana showed the highest fruit rot during the spring/ summer season as compared to the other cultivars. During the spring/ summer season, marketable yield increased with increased plant density with the highest yield obtained at 25 plants $/ \mathrm{m}^{2}$ followed by 20 plants $/ \mathrm{m}^{2}$. During the summer/fall season, plant density did not show a significant effect on total and marketable yield although a tendency for declined yield with increased density was observed. Number of fruit per plant as well as fruit size decreased with increased plant density during both seasons which did not have an effect on overall yield per plot area. Increasing the plant density to 25 plants/ $\mathrm{m}^{2}$ can improve marketable yield of determinate tomato cultivars during spring/summer season while using a plant density of 10 plants $/ \mathrm{m}^{2}$ will be more cost effective during the summer/fall season. This information will enable farmers to optimise yield and quality of determinate tomato cultivars under closed hydroponic systems.
\end{abstract}

Keywords: Solanum lycopersicum, gravel-film technique, yield, soilless cultivation.

\section{RESUMO}

Rendimento de tomate cultivado em sistema hidropônico, afetado pelo espaçamento entre plantas

$\mathrm{Na}$ África do Sul não há recomendações de espaçamento estabelecidas para a produção de cultivares de tomate em sistemas hidropônicos fechados. Determinou-se o rendimento e a qualidade de cultivares de tomate de crescimento determinado (Dynimo, Mariana, Mion e Sama) em hidroponia e densidades de plantio (10, 16, 20 e 25 plantas $/ \mathrm{m}^{2}$ ) durante primavera/verão e verão/outono. O delineamento experimental foi em blocos ao acaso, em esquema fatorial de quatro espaçamentos de plantas x quatro cultivares com três repetições. Não houve diferenças significativas no rendimento comercial entre as cultivares testadas nas duas épocas de plantio. A cultivar Mion apresentou maior incidência de podridão apical, enquanto 'Mariana' apresentou maior podridão de frutos durante a primavera/verão. Durante a primavera/verão, o rendimento comercializável aumentou com a maior densidade de plantas $\left(25 \mathrm{pl} / \mathrm{m}^{2}\right)$, seguido por $20 \mathrm{pl} / \mathrm{m}^{2}$. Durante o verão/outono, a densidade de plantas não mostrou efeito significativo no rendimento total e comercializável, embora tenha sido observada tendência de declínio com maior densidade. O número de frutos por planta bem como o tamanho do fruto diminuíram com o aumento da densidade de plantas durante as duas estações, entretanto, sem efeito no rendimento global por área de parcela. $\mathrm{O}$ aumento da densidade de plantas para $25 \mathrm{pl} / \mathrm{m}^{2}$ pode melhorar o rendimento comercializável de cultivares de tomate determinado durante a primavera/verão enquanto que a densidade de $10 \mathrm{pl} / \mathrm{m}^{2}$ será mais rentável durante o verão/outono. Esta informação permitirá aos agricultores otimizar o rendimento e a qualidade de cultivares de tomate determinado em sistemas hidropônicos fechados.

Palavras-chave: Solanum lycopersicum, técnica de cascalho, produção comercializável, produção não comercializável, cultivo sem solo.

\section{Received on January 15, 2016; accepted on November 22, 2016}

$\mathrm{T}$ omato production in South Africa is mainly under field conditions with diverse climatic conditions. Growing vegetables using soil has a range of challenges, such as variable temperatures, moisture holding capacity, available nutrient supply, proper root aeration, as well as disease and pest infestation (Maboko \& Du Plooy, 2014). Soilless production under protected conditions alleviates some of these problems, while giving the grower better control over plant growth and development (Du Plooy et al., 2012).
For successful production of tomatoes, increasing the grower's profit and satisfying the local market, requires the application of different production methods to increase yield. These include plant spacing and cultivar choice. The number of plants per unit 
area is one of the important agronomic practices to optimize yield (Rodriguez et al., 2007; Kitila et al., 2012; Maboko \& Du Plooy, 2013). An ideal plant density can lead to optimum yields, whereas too high or too low plant densities can result in relatively lower yields of poor quality (Maboko \& Du Plooy, 2013). In an optimal space, plants can efficiently utilize environmental factors, such as light, air, water and nutrients, and inter- or intra-specific competition should be at a minimum. It also ensures proper plant growth and development resulting in maximizing yield of crop and economic use of land. Manipulation of plant spacing is an important means to increase light interception and its efficient use in tomato production (Feng et al., 2010). Tomato yield has been reported to be dependent on the number of plants accommodated per unit area (Mehla et al., 2000; Belemi, 2008; Maboko et al., 2011a). Castodi et al. (2012) reported that an increase in plant density resulted in a reduction in mean fresh weight of the plants and increased productivity to a certain extent. Yield of fresh market and processing tomatoes was reported to be greatly influenced by plant spacing (Belemi, 2008). Mehla et al. (2000) also reported the importance of plant spacing on yield and quality parameters in tomato crop.

In South Africa, hydroponically grown tomatoes are generally produced in an open bag hydroponic system. However, research reports have indicated that hydroponic cultivation is inclined towards closed systems in order to reduce nutrient losses and control of the environment (Schwarz et al., 2009). A closed hydroponic system provides efficient use of water and nutrients due to recirculating of the nutrient solution. In South Africa, the gravel-film technique has shown improved yield per unit area at high density planting of tomato with decapitated growing points (Maboko \& Du Plooy, 2013). There are no established tomato spacing recommendations for determinate tomato cultivars production under a closed hydroponic system using the gravel-film technique. Cultivar selection is also a critical management decision that can impact yield and fruit quality (Maboko \& Du Plooy, 2013). The study was aimed at determining yield of hydroponically grown determinate tomato cultivars at different plant spacings.

\section{MATERIAL AND METHODS}

The study was conducted during September to December 2013 (spring/ summer season) and repeated in January to April 2014 (summer/fall season) in a $40 \%$ black and white shadenet structure at the Agricultural Research Council - Vegetable and Ornamental Plants (ARC-VOP), Roodeplaat, South Africa

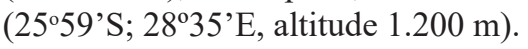

Plantlets of four tomato cultivars were transplanted 35 days after seeding using a closed (gravel-film technique) hydroponic system. A randomized complete block design in a factorial scheme of $4 \times 4$ [four planting spacings $(25 \times 40,25 \times 25,20 \times 25$ and $20 \times 20 \mathrm{~cm})$ $\mathrm{x}$ four determinate tomato cultivars (Dynimo, Mariana, Mion and Sama)], resulted in a total of 16 treatment combinations with three replicates. The planting spacings $25 \times 40,25 \times 25,20 \times 25$ and $20 \times 20 \mathrm{~cm}$ comprised respectively the following planting densities: 10 , 16,20 and 25 plants $/ \mathrm{m}^{2}$. Area occupied by a single plot was $2 \times 1 \mathrm{~m}$. Tomato seedlings were transplanted $6 \mathrm{~cm}$ deep into gullies ( $17 \mathrm{~m}$ long $\mathrm{x} 1 \mathrm{~m}$ wide) filled to a depth of $6 \mathrm{~cm}$ with crushed granite rocks of irregular shape, with a diameter

Table 1. Effect of plant density on yield and fruit size of determinate tomato cultivars (spring/summer season). South Africa, Agricultural Research Council, 2014.

\begin{tabular}{|c|c|c|c|c|c|c|c|}
\hline Treatment & $\begin{array}{c}\text { Total yield } \\
\left(\mathrm{kg} / \mathrm{m}^{2}\right)\end{array}$ & $\begin{array}{c}\text { Total fruits } \\
\text { (number } / \mathbf{m}^{2} \text { ) }\end{array}$ & $\begin{array}{c}\text { Marketable } \\
\text { yield } \\
\left(\mathbf{k g} / \mathbf{m}^{2}\right) \\
\end{array}$ & $\begin{array}{c}\text { Marketable } \\
\text { fruits } \\
\text { (number/m²) }\end{array}$ & $\begin{array}{c}\text { Unmarketable } \\
\text { yield } \\
\left(\mathbf{k g} / \mathbf{m}^{2}\right) \\
\end{array}$ & $\begin{array}{c}\text { Extra-small } \\
\text { fruits } \\
\text { (number/m²) }\end{array}$ & $\begin{array}{c}\text { Extra-small } \\
\text { fruits } \\
\left(\mathrm{kg} / \mathrm{m}^{2}\right) \\
\end{array}$ \\
\hline \multicolumn{8}{|l|}{$\begin{array}{l}\text { Density } \\
\left(\text { plants } / \mathbf{m}^{2}\right)\end{array}$} \\
\hline 10 & $15.8 \mathrm{c}$ & $231.7 \mathrm{c}$ & $12.5 \mathrm{c}$ & $140.7 b$ & $3.3 b$ & $78.7 \mathrm{c}$ & $2.3 \mathrm{c}$ \\
\hline 16 & $24.6 \mathrm{~b}$ & $359.7 \mathrm{~b}$ & $19.6 \mathrm{~b}$ & $212.6 \mathrm{a}$ & $5.0 \mathrm{ab}$ & $128.3 b$ & $3.8 \mathrm{~b}$ \\
\hline 20 & $25.2 \mathrm{~b}$ & $389.9 \mathrm{ab}$ & $19.7 \mathrm{ab}$ & $226.2 \mathrm{a}$ & $5.5 \mathrm{a}$ & $146.8 \mathrm{ab}$ & $4.4 \mathrm{ab}$ \\
\hline 25 & $29.7 \mathrm{a}$ & $431.5 \mathrm{a}$ & $23.3 \mathrm{a}$ & $252.4 \mathrm{a}$ & $6.4 \mathrm{a}$ & $151.2 \mathrm{a}$ & $4.8 \mathrm{a}$ \\
\hline LSD 0.05 & 3.8 & 51.17 & 3.4 & 40.08 & 0.7 & 22.07 & 0.63 \\
\hline \multicolumn{8}{|l|}{ Cultivars } \\
\hline Dynimo & $26.0 \mathrm{a}$ & $379.5 \mathrm{a}$ & 19.6 & $206.6 \mathrm{ab}$ & $6.4 \mathrm{a}$ & $154.5 \mathrm{a}$ & $5.2 \mathrm{a}$ \\
\hline Mariana & $25.8 \mathrm{ab}$ & $408.2 \mathrm{a}$ & 19.6 & $231.6 \mathrm{a}$ & $6.1 \mathrm{a}$ & $149.2 \mathrm{a}$ & $4.2 b$ \\
\hline Mion & $22.2 b c$ & $398.2 \mathrm{a}$ & 17.0 & $226.2 \mathrm{a}$ & $5.2 b$ & $149.5 \mathrm{a}$ & $4.0 \mathrm{~b}$ \\
\hline Sama & $21.3 \mathrm{c}$ & $226.9 b$ & 18.8 & $167.1 \mathrm{~b}$ & $2.5 \mathrm{c}$ & $51.8 \mathrm{~b}$ & $1.9 \mathrm{c}$ \\
\hline LSD 0.05 & 3.8 & 51.17 & ns & 40.08 & 0.7 & 22.07 & 0.63 \\
\hline
\end{tabular}

Values in a column followed by the same letter are not significantly different ( $\mathrm{p}>0.05)$, using Fishers' protected t-test; LSD: least significant difference. 
ranging from 16 to $19 \mathrm{~mm}$. A gravel-film technique hydroponic system was used to perform the trial, as described by Maboko et al. (2011b). Plants were not trellised or pruned.

The nutrient solution was renewed on a weekly basis. The composition and chemical concentration of fertilizers used in the nutrient solution were: Hygroponic $^{\circledR}$ (Hygrotech (Pty). Ltd, South Africa) comprising of N (68 ppm), $\mathrm{P}$ (42 ppm), K (208 ppm), Mg (30 ppm), $\mathrm{S}$ (64 ppm), Fe (1.254 ppm), Cu (0.022 ppm), Zn (0.149 ppm), Mn (0.299 ppm), B (0.373 ppm), and Mo (0.037 ppm), as well as calcium nitrate $\left[\mathrm{Ca}\left(\mathrm{NO}_{3}\right)_{2}\right]$ comprising of $\mathrm{N}$ (117 ppm) and $\mathrm{Ca}$ (166 ppm). The fertilizers applied from transplanting until the plants were three weeks old were $600 \mathrm{~g}$ Hygroponic and $600 \mathrm{~g} \mathrm{Ca}\left(\mathrm{NO}_{3}\right)_{2}$ in $1000 \mathrm{~L}$ water. Thereafter, $900 \mathrm{~g}$ Hygroponic and $900 \mathrm{~g}$ calcium nitrate were applied per $1000 \mathrm{~L}$ water. The electrical conductivity (EC) and $\mathrm{pH}$ of the nutrient solution were maintained within a range of 1.9 to 2.3 $\mathrm{mS} / \mathrm{cm}$ and 5.8 to 6.1 , respectively.

Fruits were harvested on a weekly basis at breaker stage. The performance of the tomato cultivars was evaluated for total yield, marketable and unmarketable yield, as well as the occurrence of physiological disorders. Fruits were regarded as unmarketable when they exhibited cracking, zippering, rotting, blossom-end rot, rain-check, cat-face or fell into the extra small size category (less than $40 \mathrm{~mm}$ diameter). Marketable yield comprised of fruits that were larger than $40 \mathrm{~mm}$ diameter, whereas total yield was determined by adding marketable yield and unmarketable yield.

Data were subjected to analysis of variance (ANOVA) using the statistical program GenStat ${ }^{\circledR}$ version 11.1 (Payne et al., 2008). Treatment means were separated using Fisher's protected T-test least significant difference (LSD) at the $5 \%$ level of significance (Snedecor \& Cochran, 1980).

\section{RESULTS AND DISCUSSION}

High mass of extra-small sized fruits were found at 20 and 25 plants/ $\mathrm{m}^{2}$, although plants $/ \mathrm{m}^{2}$ did not differ significantly with 16 plants $/ \mathrm{m}^{2}$ (Table 1). A close spacing (20 to 25 plants/ $\mathrm{m}^{2}$ ) during the spring/summer season resulted in more small fruits of unmarketable size (Table 1) which could be explained most likely by a decrease in plant growth rate and inadequate supply of photosynthates due to shading and reduced light interception per plant (Heuvelink, 1995; Papadopoulos \& Pararajasingham, 1997). The lower leaves were observed to be gradually shaded by the canopy especially at high plant density where

shading progressively decreases the light intensity (Van Henten et al., 2002). Trouwborst et al. (2011) reported that shading of developed and matured leaves dramatically decreased leaf photosynthetic capacity and leaf chlorophyll content within a few days. Plants grown at closer spacing (20 to 25 plants $/ \mathrm{m}^{2}$ ) grew taller with thinner stems than widely spaced plants (10 to 16 plants $/ \mathrm{m}^{2}$ ) while lower leaves became yellowish as a result of shading. Tomato yield is determined primarily by the amount of intercepted light (Newton et al., 1999) and assimilate partitioning (Ho, 1996).

Table 2. Effect of determinate tomato cultivars at different plant densities on tomato yield per plant during spring/summer season and summer/fall season. South Africa, Agricultural Research Council, 2014.

\begin{tabular}{|c|c|c|c|c|}
\hline Treatment & $\begin{array}{l}\text { Total fruits } \\
\text { (number/ } \\
\text { plant) }\end{array}$ & $\begin{array}{c}\text { Total } \\
\text { yield } \\
\text { (kg/plant) }\end{array}$ & $\begin{array}{c}\text { Marketable } \\
\text { yield } \\
\text { (kg/plant) }\end{array}$ & $\begin{array}{c}\text { Marketable } \\
\text { fruits } \\
\text { (num./plant) }\end{array}$ \\
\hline Density (plants/m²) & \multicolumn{4}{|c|}{ Spring/summer season } \\
\hline 10 & $23.2 \mathrm{a}$ & $1.58 \mathrm{a}$ & $1.25 \mathrm{a}$ & $14.1 \mathrm{a}$ \\
\hline 16 & $22.5 \mathrm{a}$ & $1.54 \mathrm{a}$ & $1.23 \mathrm{a}$ & $13.3 \mathrm{a}$ \\
\hline 20 & $19.5 \mathrm{ab}$ & $1.26 \mathrm{~b}$ & $0.99 b$ & $11.3 \mathrm{ab}$ \\
\hline 25 & $17.3 b$ & $1.19 \mathrm{~b}$ & $0.93 b$ & $10.1 b$ \\
\hline LSD 0.05 & 3.79 & 0.25 & 0.23 & 2.85 \\
\hline \multicolumn{5}{|l|}{ Cultivar } \\
\hline Dynimo & $21.9 \mathrm{a}$ & 1.49 & 1.14 & 12.2 \\
\hline Mariana & $24.1 \mathrm{a}$ & 1.54 & 1.16 & 13.5 \\
\hline Mion & $23.0 \mathrm{a}$ & 1.25 & 0.96 & 13.1 \\
\hline Sama & $13.6 \mathrm{~b}$ & 1.28 & 1.14 & 10.0 \\
\hline LSD 0.05 & 3.79 & $\mathrm{~ns}$ & ns & $\mathrm{ns}$ \\
\hline Density (plants $/ \mathbf{m}^{2}$ ) & \multicolumn{4}{|c|}{ Summer/fall season } \\
\hline 10 & $28.4 \mathrm{a}$ & $2.20 \mathrm{a}$ & $1.96 \mathrm{a}$ & $23.3 \mathrm{a}$ \\
\hline 16 & $18.9 b$ & $1.40 \mathrm{~b}$ & $1.23 b$ & $15.1 b$ \\
\hline 20 & $14.5 \mathrm{c}$ & $1.03 \mathrm{c}$ & $0.89 \mathrm{c}$ & $11.3 \mathrm{c}$ \\
\hline 25 & $10.6 \mathrm{~d}$ & $0.76 \mathrm{~d}$ & $0.65 \mathrm{c}$ & $8.3 \mathrm{c}$ \\
\hline LSD 0.05 & 3.67 & 0.27 & 0.25 & 3.16 \\
\hline \multicolumn{5}{|l|}{ Cultivar } \\
\hline Dynimo & $17.2 \mathrm{ab}$ & 1.26 & 1.078 & 13.5 \\
\hline Mariana & $20.4 \mathrm{a}$ & 1.38 & 1.181 & 15.5 \\
\hline Mion & 20.1a & 1.39 & 1.166 & 15.1 \\
\hline Sama & $14.7 \mathrm{~b}$ & 1.34 & 1.296 & 13.9 \\
\hline LSD 0.05 & 3.67 & ns & ns & $\mathrm{ns}$ \\
\hline
\end{tabular}

Values in a column followed by the same letter are not significantly different ( $p>0.05)$, using Fishers' protected t-test: least significant difference. 
During the spring/summer season, cultivars planted at a density of 25 plants $/ \mathrm{m}^{2}$ produced significantly higher total yield while those planted at 10 plants $/ \mathrm{m}^{2}$ produced the lowest yield (Table 1). Marketable yield was higher at plant densities of 25 and 20 plants/ $\mathrm{m}^{2}$, although 20 plants $/ \mathrm{m}^{2}$ did not differ significantly from 16 plants $/ \mathrm{m}^{2}$. During the spring/summer season, number of marketable and unmarketable fruits $/ \mathrm{m}^{2}$ were significantly higher at 16,20 and 25 plants $/ \mathrm{m}^{2}$ compared to 10 plants/ $\mathrm{m}^{2}$ (Table 1). Total number of fruits and number of marketable fruits per plant were high at 10 and 16 plants $/ \mathrm{m}^{2}$, although not significantly different from 20 plants $/ \mathrm{m}^{2}$ density (Table 2 ). A wider plant spacing of 10 and 16 plants $/ \mathrm{m}^{2}$ produced significantly higher total yield and marketable yield per plant than 20 and 25 plants $/ \mathrm{m}^{2}$ (Table 2). Tomato yield is directly related to the number of plants per unit area, number of harvested fruits per plant and average fruit mass (Streck et al., 1998). During the summer/fall season, total number of fruits, total yield, marketable yield and number of marketable fruits per plant decreased with an increase in plant density (Table 2). In contrast, during the summer/fall season the yield and yield components per unit area were not affected by plant density (Table 3 ). However, there was a decreased tendency in total and marketable yield with an increase in plant density (Table 3). Furthermore, effect of plant density was influenced by growing season/environmental conditions. An increase in plant density (Table 1) resulted in an increase in total yield during the spring/summer season while in summer/fall season plant density did not have a significant effect (Table 3). During the spring/ summer season, there was an increase in marketable yield with an increase in plant density (Table 1) whereas during the summer/fall season (Table 3) marketable yield increased with a decrease in plant density. Growers are only interested in high marketable yield to generate profit.

Generally, determinate tomato does not require sucker/stem pruning, and if pruned, the yield will be reduced. Each shoot of a determinate tomato results in a cluster/flower truss and consequently

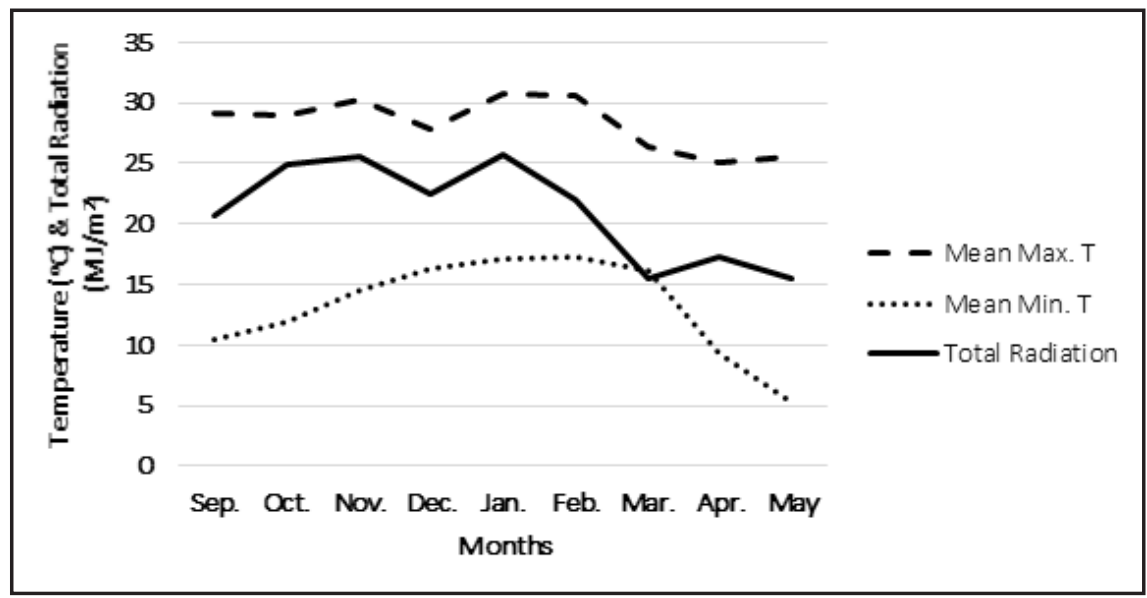

Figure 1. Temperature and total radiation conditions during the experimental period outside the shadenet structure (condições de temperatura e radiação total durante o experiment, fora da casa de vegetação). South Africa, Agricultural Research Council, 2014.

Table 3. Effect of plant density on yield and fruit size of determinate tomato cultivars (summer/fall season). South Africa, Agricultural Research Council, 2014.

\begin{tabular}{|c|c|c|c|c|c|c|c|}
\hline Treatment & $\begin{array}{c}\text { Total yield } \\
\left(\mathrm{kg} / \mathrm{m}^{2}\right)\end{array}$ & $\begin{array}{c}\text { Total fruits } \\
\left(\text { number } / \mathbf{m}^{2} \text { ) }\right.\end{array}$ & $\begin{array}{c}\text { Marketable } \\
\text { yield } \\
\left(\mathrm{kg} / \mathrm{m}^{2}\right)\end{array}$ & $\begin{array}{c}\text { Marketable } \\
\text { yield } \\
\text { (number } / \mathbf{m}^{2} \text { ) }\end{array}$ & $\begin{array}{c}\text { Unmarketable } \\
\text { yield } \\
\left(\mathbf{k g} / \mathbf{m}^{2}\right)\end{array}$ & $\begin{array}{c}\text { Extra-small } \\
\text { fruits } \\
\text { (number } / \mathrm{m}^{2} \text { ) }\end{array}$ & $\begin{array}{c}\text { Extra-small } \\
\text { fruits } \\
\left(\mathrm{kg} / \mathrm{m}^{2}\right)\end{array}$ \\
\hline \multicolumn{8}{|c|}{ Density (plants/m²) } \\
\hline 10 & 22.0 & 284 & 19.6 & 233.0 & 2.4 & 47.0 & 2.1 \\
\hline 16 & 22.4 & 303 & 19.7 & 242.2 & 2.7 & 54.6 & 2.3 \\
\hline 20 & 20.5 & 289 & 17.7 & 226.8 & 2.8 & 56.9 & 2.4 \\
\hline 25 & 18.9 & 264 & 16.1 & 206.3 & 2.7 & 53.0 & 2.3 \\
\hline LSD 0.05 & ns & ns & ns & ns & ns & ns & ns \\
\hline \multicolumn{8}{|l|}{ Cultivar } \\
\hline Dynimo & 20.1 & 276 & 17.0 & 214.6 & $3.1 \mathrm{a}$ & $55.3 b$ & $2.6 \mathrm{~b}$ \\
\hline Mariana & 21.5 & 320 & 18.2 & 241.8 & $3.3 \mathrm{a}$ & $72.5 \mathrm{a}$ & $2.9 \mathrm{ab}$ \\
\hline Mion & 20.8 & 305 & 17.3 & 225.4 & $3.6 \mathrm{a}$ & $73.5 \mathrm{a}$ & $3.2 \mathrm{a}$ \\
\hline Sama & 21.4 & 239 & 20.7 & 226.5 & $0.7 \mathrm{~b}$ & $10.2 \mathrm{c}$ & $0.5 \mathrm{c}$ \\
\hline LSD 0.05 & ns & ns & ns & ns & 0.57 & 11.89 & 0.49 \\
\hline
\end{tabular}

Values in a column followed by the same letter are not significantly different $(\mathrm{p}>0.05)$, using Fishers' protected t-test; ns $=$ not significant LSD: least significant difference. 
Table 4. Effect of plant density on fruit physiological disorders of determinate tomato cultivars (spring/summer season). South Africa, Agricultural Research Council, 2014.

\begin{tabular}{|c|c|c|c|c|c|c|}
\hline \multirow[t]{2}{*}{ Treatment } & \multicolumn{2}{|c|}{ Fruit cracking } & \multicolumn{2}{|c|}{ Blossom-end rot } & \multicolumn{2}{|c|}{ Cat-face } \\
\hline & (number/m $\left.\mathbf{m}^{2}\right)$ & $\operatorname{Mass}\left(\mathrm{g} / \mathrm{m}^{2}\right)$ & (number/m²) & Mass $\left(\mathrm{g} / \mathrm{m}^{2}\right)$ & (number/m $\left.\mathbf{m}^{2}\right)$ & $\operatorname{Mass}\left(\mathrm{g} / \mathrm{m}^{2}\right)$ \\
\hline \multicolumn{7}{|c|}{ Density (plants $/ \mathbf{m}^{2}$ ) } \\
\hline 10 & 0.0 & 0.9 & $0.3 \mathrm{c}$ & $10.7 \mathrm{~b}$ & 0.0 & 0.3 \\
\hline 16 & 0.1 & 12.5 & $1.6 \mathrm{a}$ & $61.5 \mathrm{a}$ & 0.0 & 6.3 \\
\hline 20 & 0.0 & 0.0 & $0.3 \mathrm{c}$ & $13.6 \mathrm{~b}$ & 0.1 & 14.5 \\
\hline 25 & 0.2 & 12.6 & $0.9 \mathrm{~b}$ & $56.7 \mathrm{a}$ & 0.1 & 17.7 \\
\hline LSD 0.05 & ns & ns & 0.57 & 27.58 & $\mathrm{~ns}$ & $\mathrm{~ns}$ \\
\hline \multicolumn{7}{|l|}{ Cultivar } \\
\hline Dynimo & 0.2 & 16.1 & $0.5 b$ & $22.1 \mathrm{bc}$ & 0.2 & 27.6 \\
\hline Mariana & 0.0 & 1.7 & $0.2 \mathrm{~b}$ & $11.6 \mathrm{c}$ & 0.0 & 0.5 \\
\hline Mion & 0.1 & 8.2 & $1.8 \mathrm{a}$ & $59.7 \mathrm{a}$ & 0.0 & 0.0 \\
\hline Sama & 0.0 & 0.0 & $0.7 \mathrm{~b}$ & $49.0 \mathrm{ab}$ & 0.1 & 10.5 \\
\hline \multirow[t]{3}{*}{ LSD 0.05} & ns & ns & 0.57 & 27.58 & $\mathrm{~ns}$ & ns \\
\hline & \multicolumn{2}{|c|}{ Fruit rot } & \multicolumn{2}{|c|}{ Zippering } & & \\
\hline & (number/m²) & $\operatorname{Mass}\left(\mathrm{g} / \mathrm{m}^{2}\right)$ & (number/m²) & $\operatorname{Mass}\left(\mathrm{g} / \mathrm{m}^{2}\right)$ & & \\
\hline \multicolumn{5}{|c|}{ Density (plants $/ \mathbf{m}^{2}$ ) } & & \\
\hline 10 & $4.8 \mathrm{~b}$ & 626 & $6.2 b$ & $351 b$ & & \\
\hline 16 & $11.5 \mathrm{a}$ & 713 & $5.8 \mathrm{~b}$ & $421 \mathrm{~b}$ & & \\
\hline 20 & $11.3 \mathrm{a}$ & 673 & $5.4 \mathrm{~b}$ & $404 b$ & & \\
\hline 25 & $15.2 \mathrm{a}$ & 838 & $11.5 \mathrm{a}$ & $651 \mathrm{a}$ & & \\
\hline LSD 0.05 & 4.75 & ns & 4.1 & 202.7 & & \\
\hline \multicolumn{7}{|l|}{ Cultivar } \\
\hline Dynimo & $9.5 b$ & $616 b$ & $7.0 \mathrm{ab}$ & $471 \mathrm{~b}$ & & \\
\hline Mariana & $16.1 \mathrm{a}$ & $1204 a$ & $10.9 \mathrm{a}$ & $690 \mathrm{a}$ & & \\
\hline Mion & $13.4 \mathrm{ab}$ & $721 \mathrm{~b}$ & $7.3 \mathrm{ab}$ & $352 b$ & & \\
\hline Sama & $3.9 \mathrm{c}$ & $310 \mathrm{~b}$ & $3.7 \mathrm{~b}$ & $314 \mathrm{~b}$ & & \\
\hline LSD 0.05 & 4.75 & 441.1 & 4.1 & 202.7 & & \\
\hline
\end{tabular}

Values in a column followed by the same letter are not significantly different ( $p>0.05)$, using Fishers' protected t-test; ns $=$ not significant LSD: least significant difference.

fruit cluster/fruit truss. It was observed that plants grown at the wider spacing (10 and 16 plants $/ \mathrm{m}^{2}$ ) exhibited more side shoots and continuous vegetative growth due to less population pressure per unit area which resulted in similar marketable yield to plants grown at a higher population density (20 and 25 plants $/ \mathrm{m}^{2}$ ). Wide spacing might have an advantage of optimum conditions for vegetative growth and better plant canopy due to maximum light interception, photosynthesis, assimilation and accumulation of more photosynthates into the plant system (Mazumdar et al., 2007). This was evident from the increase in number of fruits, total yield and marketable yield per plant at a wider spacing of 10 and 16 plants $/ \mathrm{m}^{2}$ (Table 2). Increased total yield at high density planting can be attributed to the high number of plants/ $\mathrm{m}^{2}$ (Table 1). During the summer/fall season, plants were exposed to shorter days, lower temperature and radiation, which might have not fully benefited densely planted plants as a result of competition for light (Figure 1). Increase in total number of fruits, total yield and marketable yield per plant as well as increased tendency of tomato yield/ $\mathrm{m}^{2}$ with a decrease in plant density during summer/fall season (Tables 2 and 3) as opposed to spring/summer season (Tables 1 and 2) could also be due to shorter day length and lower radiation (Figure 1). We observed that plants tended to produce fewer flowers at closer spacing which contributed to lower yield compared to wider spacing during summer/fall season. Temperature and radiation during spring/summer season might have been sufficient to benefit closer spaced plants to produce high yield (Figure 1).

Plants grown at 16 or 25 plants/ $\mathrm{m}^{2}$ produced more fruits exhibiting blossom-end rot (Table 4). Number 
Table 5. Effect of plant density on fruit physiological disorders of determinate tomato cultivars (summer/fall season). South Africa, Agricultural Research Council, 2014.

\begin{tabular}{|c|c|c|c|c|c|c|}
\hline \multirow{2}{*}{ Treatment } & \multicolumn{2}{|c|}{ Cat-face } & \multicolumn{2}{|c|}{ Fruit rot } & \multicolumn{2}{|c|}{ Zippering } \\
\hline & (number/m²) & $\operatorname{Mass}\left(\mathrm{g} / \mathrm{m}^{2}\right)$ & (number/m²) & $\operatorname{Mass}\left(\mathrm{g} / \mathrm{m}^{2}\right)$ & (number/m²) & $\operatorname{Mass}\left(\mathrm{g} / \mathrm{m}^{2}\right)$ \\
\hline \multicolumn{7}{|c|}{ Density (plants/m²) } \\
\hline 10 & 0.00 & 0.0 & 3.54 & 254 & 0.83 & 76 \\
\hline 16 & 0.00 & 0.0 & 5.58 & 357 & 0.58 & 55 \\
\hline 20 & 0.04 & 5.6 & 4.42 & 316 & 0.96 & 76 \\
\hline 25 & 0.00 & 0.0 & 3.50 & 286 & 1.46 & 115 \\
\hline LSD 0.05 & $\mathrm{~ns}$ & $\mathrm{~ns}$ & ns & ns & $\mathrm{ns}$ & $\mathrm{ns}$ \\
\hline \multicolumn{7}{|l|}{ Cultivar } \\
\hline Dynimo & 0.00 & 0.0 & $5.04 \mathrm{a}$ & $404 a$ & 1.21 & 105 \\
\hline Mariana & 0.00 & 0.0 & $5.00 \mathrm{a}$ & $272 \mathrm{ab}$ & 1.12 & 77 \\
\hline Mion & 0.00 & 0.0 & $5.17 \mathrm{a}$ & $355 a$ & 0.88 & 64 \\
\hline Sama & 0.04 & 5.6 & $1.83 \mathrm{~b}$ & $181 \mathrm{~b}$ & 0.62 & 77 \\
\hline LSD 0.05 & ns & ns & 2.12 & 142 & ns & ns \\
\hline
\end{tabular}

Values in a column followed by the same letter are not significantly different ( $p>0.05)$, using Fishers' protected t-test; ns= not significant LSD: least significant difference.

of fruit rot was significantly higher at plant density of 16,20 or 25 plants/ $\mathrm{m}^{2}$. A closer spacing of 25 plants $/ \mathrm{m}^{2}$ produced significantly higher incidence of fruit zippering (Table 4). High density planting $\left(16,20\right.$ and 25 plants $\left./ \mathrm{m}^{2}\right)$ created problems of phyto-sanitation due to moisture accumulation and poor ventilation at the bottom of the plants, which facilitated fruit rot (Table 4). High incidence of zippering was found at high plant density which could be due to poor ventilation or pollination. This is in agreement with the study conducted by Maboko \& Du Plooy (2013). Zippering is a physiological disorder whereby an anther becomes attached to the ovary wall of the newly forming fruit causing zipper to form. Plant density did not have a significant effect on fruit physiological disorders during summer/ fall season (Table 5).

During the spring/summer season, the highest total yield was obtained by Dynimo and Mariana, although Mariana did not differ significantly from Mion (Table 1). Total number of fruits was significantly higher for Dynimo, Mariana (control) and Mion compared to Sama. Marketable yield was not affected by cultivar choice, however, number of marketable fruits was higher for Dynimo, Mariana and Mion (Table
1). Dynimo and Mariana produced significantly higher unmarketable yield followed by Mion and the least was Sama. Dynimo and Mariana were the cultivars with the highest total yield. However, marketable yield of all cultivars was similar, due to more unmarketable fruits in Dynimo and Mariana (Table 1). Mion had the highest incidence of blossom-end rot, although did not differ significantly from Sama (Table 4). Mariana was more susceptible to incidence of fruit rot and zippering compared to Dynimo, Mion and Sama (Table 4).

During spring/summer season, there were no significant differences in total yield, marketable yield and number of marketable fruits per plant in all cultivars with the exception of lower total number of fruits for Sama (Table 2). Furthermore, there were no differences in total yield, total number of fruits, marketable yield and number of marketable fruits $/ \mathrm{m}^{2}$ amongst the four cultivars (Table 3 ). However, Sama produced the smallest unmarketable yield and mass of extra-small sized fruits compared to Dynimo, Mariana and Mion. Similar to results obtained in spring/summer season, extra-small sized fruits contributed to high unmarketable yield for cultivars Dynimo, Mariana and Mion (Table 3). Generally, Dynimo produced a small number of fruits (Tables 1 and 2), but larger sized fruits. Results showed that spring/ summer season cultivars produced higher unmarketable yield and total yield than summer/fall growing season. Incidence of cat-face and zippering were not affected by cultivar, however, fruit rots were high for cultivars Dynimo, Mariana and Mion during summer/fall season (Table 5). When selecting tomato cultivars, growers should consider the market demand, fruit characteristics as well as the yielding potential and resistance to diseases. Mariana was more susceptible to incidence of fruit rot and zippering compared to Dynimo, Mion and Sama which contributed to high unmarketable yield. Although, Sama produced fruits with the least unmarketable yield and number of marketable fruits, it performed similarly to other cultivars in terms of marketable yield. The reason is due to the larger sized fruits produced by this cultivar, Sama. High incidence of extra-small sized fruits (Table 1) contributed to high unmarketable yield among the cultivars.

Results indicate that a plant density of 20 plants $/ \mathrm{m}^{2}$ can improve marketable yield of determinate tomato cultivars during spring/summer season in closed 
hydroponic system using gravel-film technique. However, during summer/ fall season a plant density of 10 plants/ $\mathrm{m}^{2}$ was most cost effective, with no significant increase in yield at higher densities. Prior to this study there was no standard plant density recommendation available for determinate tomato cultivars in a closed (gravel) hydroponic system. This information will enable farmers to optimise yield and quality of determinate tomato cultivars under closed hydroponic systems.

\section{REFERENCES}

BELEMI, T. 2008. Response of tomato cultivars differing in growth habit to nitrogen and phosphorus fertilisers and spacing on vertisol in Ethiopia. Acta Agriculturae Slovenica 91: 103-119.

CASTOLDI, R; FAVERI, LA; SOUZA, JO; BRAZ, LT; CHARLO, HCO. 2012. Productivity characteristics of endive as a function of spacing. Acta Horticulturae 936 : 305-310.

DU PLOOY, CP; MABOKO, MM; VAN DEN HEEVER, E, CHILOANE, S. 2012. Research and technology transfer by Agricultural Research Council to sustain South African hydroponic industry. Acta Horticulturae 947: 147-151.

FENG, H; ZHANG, T; SHI, YT; WANG, WJ; WANG, WH. 2010. Research of plant type and light distribution of tomatoes determined by imaging technology. African Journal of Agricultural Research 5: 1860-1867.

HEUVELINK, E. 1995. Effect of plant density on biomass allocation to the fruits in tomato
(Lycopersicon esculentum Mill.). Scientia Horticulturae 64: 193-201.

HO, LC. 1996. The mechanism of assimilate partitioning and carbohydrate compartmentation in fruit in relation to the quality and yield of tomato. Journal of Experimental Botany 47: 1239-1243.

KITILA, M; BELEW, D; MOHAMMED, A; GETACHEW, Y. 2012. Effect of intra-row spacing and variety of fruit yield and quality of fresh market tomato (Lycopersicon esculentum Mill.) under Jimma condition, Ethiopia. Ethiopian Journal of Applied Sciences and Technology 3: 32-42.

MABOKO, MM; DU PLOOY, CP. 2013. Highdensity planting of tomato cultivar's with early decapitation of growing point increased yield in a closed hydroponic system. Acta Agriculturae Scandinavica 63: 676-682.

MABOKO, MM; DU PLOOY, CP. 2014. Yield of hydroponically grown tomato cultivars as affected by transplanting stage or direct seeding. HortScience 49: 438-440.

MABOKO, MM, DU PLOOY, CP; BERTLING, I. $2011 \mathrm{~b}$. Comparative performance of tomato cultivars cultivated in two hydroponic production systems. South African Journal of Plant and Soil 28: 97-102.

MABOKO, MM; DU PLOOY, CP; CHILOANE, S. 2011a. Effect of plant population, stem and fruit pruning of hydroponically grown tomato. African Journal of Agricultural Research 6: 5144-5148.

MAZUMDAR, SN; MONINUZZAMAN, M; RAHMAN, SMM; BASAK, NC. 2007. Influence of support systems and spacing on hyacinth bean production in the eastern hilly area of Bangladesh. Legume Research 30: 1-9.

MEHLA, CP; SRIVASTAVA, VK, JAGE, S; MANGAT, R, SINGH, J; RAM, M. 2000. Response of tomato varieties to $\mathrm{N} \& \mathrm{P}$ fertilization and spacing. Indian Journal of Agricultural Research 34: 182-184.
NEWTON, P; SAHRAOUI, R; ECONOMARKS C. 1999. The influence of air temperature on truss weight of tomatoes. Acta Horticulturae 507: 43-49.

PAPADOPOULOS, AP; PARARAJASINGHAM, S. 1997. The influence of plant spacing on light interception and use in greenhouse tomato (Lycopersicon esculentum Mill.): A review. Scientia Horticulturae 69: 1-29.

PAYNE, RW; MURRAY, DA; HARDING, SA; BAIRD, DB; SOUTAR, DM. 2008. GenStat for Windows ${ }^{\circledR}\left(11^{\text {th }}\right.$ ed) Introduction. Hemel Hempstead: VSN International.

RODRIGUEZ, JC; SHAW, NL; CANTLIFFE, DJ. 2007. Influence of plant density on yield and fruit quality of greenhouse-grown galia muskmelons. HortTechnology 17: 580-585.

SCHWARZ, D; FRANKEN, P; KRUMBEIN, A; KLÄRING, HP; BAR-YOSEF, B. 2009. Nutrient management in soilless culture in the conflict of plant, microorganism, consumer and environmental demands. Acta Horticulturae 843: 27-34.

SNEDECOR, GW; COCHRAN, WG. 1980. Statistical methods ( $\left.7^{\text {th }} \mathrm{ed}\right)$, USA: Iowa State University Press.

STRECK, NA; BURIOL, GA; ANDRIOLO, JL; SANDRI, MA. 1998. Influência da densidade de plantas e da poda apical drástica na produtividade do tomateiro em estufa de plástico. Pesquisa Agropecuária Brasileira 33: 1105-1112.

TROUWBORST, G; HOGEWONING, SW; HARBINSON, J; IEPEREN, WV. 2011. The influence of light intensity and leaf age on the photosynthetic capacity of leaves within a tomato canopy. Journal of Horticultural Science and Biotechnology 86: 403-407.

VAN HENTEN, EJ; HEMMING, J; VAN TUIJL, BAJ; MEULEMAN, J; BONTSEMA, J; VAN OS, EA. 2002. An autonomous robot for harvesting cucumbers in greenhouses. Autonomous Robots 13: 241-258. 